\title{
EXERCITATIONIS GRAMMATICAE SPECIMINA
}

\section{FRANCISCO BVECHELERO DOCTORI MAGISTROQVE BENEMERENTISSIMO}

SEMINARII PHILOLOGORVM BONNENSIS

$$
\text { SODALES }
$$

DOCTORIS NOMEN MVNVSQVE

ANTE HAEC QVINQVE LVSTRA CONLATVM

$$
\text { GRATVLATVRI }
$$

$\mathrm{D} \cdot \mathrm{D} \cdot \mathrm{L} \cdot \mathrm{M}$

A $\cdot$ D $\cdot$ III $\cdot$ ID $\cdot$ MART $\cdot A \cdot C I O I J C C C L X X X I$ 
BONNAE TYPIS EXPRESSIT CAROLVS GEORGI VNIV. TYP. 
GRAMMATICAE DOCTOR LVSTRIS NVNC QVINQVE PROBATE VOTA FACIT PRO TE FIDA CATERVA TVA

GRATA VTINAM HAEC TIBI SINT STVDII QVAE PRIMA SACRAMVS PIGNORA NON PRETIO SED PIETATE TAMEN

FriDERICVS MARX

Petrvis Sonnenbvrg

HENRICYS WOLF

Edvardvs Anspach

PavLvS Wolters

GVILELMVS LARFELD

HermanNvS HOEFER

FRIDERICVS KOEPP

FERDinandvs DvemMleR

FRIDERICVS MARCKS

ARTHVR FREDERKING 
